\title{
Instability of hypersonic boundary layer on a wall with resonating micro-cavities
}

\author{
Alexander V. Fedorov ${ }^{*}$ \\ Moscow Institute of Physics and Technology, Zhukovsky, 140180, Russia \\ Guillaume A. Brès, ${ }^{\dagger}$ Matthew Inkman, ${ }^{\ddagger}$ and Tim Colonius ${ }^{\S}$ \\ California Institute of Technology, Pasadena, CA 91125, U.S.A.
}

\begin{abstract}
Ultrasonically absorptive coatings (UAC) can stabilize the Mack second mode and thereby increase the laminar run on configurations where laminar-turbulent transition is second-mode dominated. Theory indicates that the stabilization effect can be essentially enhanced by increasing the UAC porosity. However, direct numerical simulations (DNS) showed that coatings having closely spaced grooves can trigger a new instability whose growth rate can be larger than that of Mack' second mode. The nature of the new instability is investigated theoretically and numerically. 2D linear DNS and stability analysis are performed for the temporally evolving boundary layer on a flat wall at the outer-flow Mach number 6. The wall is covered by UAC comprising equally-spaced spanwise grooves. It is shown that the new mode is associated with acoustic resonances in the grooves. Disturbance fields near mouths of resonating cavities are coupled such that the boundary-layer disturbance is decelerated and becomes unstable. To avoid this detrimental effect the coating should have sufficiently small porosity and/or narrow pores of sufficiently small aspect ratio. Restrictions on these parameters can be estimated using the linear stability theory with the impedance boundary conditions.
\end{abstract}

\section{Nomenclature}

$\begin{array}{lll}A & =\text { porous-layer admittance } \\ A r=2 b^{*} / H^{*} & =\text { cavity aspect ratio } \\ b & =\text { cavity half-width } \\ c & =\text { complex phase speed } \\ f & =\text { frequency } \\ H & =\text { cavity depth or UAC thickness } \\ M & =\text { Mach number } \\ p & =\text { pressure disturbance amplitude } \\ \operatorname{Re}=H^{*} \rho_{e}^{*} U_{e}^{*} / \mu_{e}^{*} & =\text { Reynolds number based on cavity depth } \\ s & =\text { cavity spacing } \\ T & =\text { mean flow temperature } \\ u, v, w & =\text { components of velocity disturbance } \\ x, y, z & =\text { Cartesian coordinates } \\ \alpha, \beta & =\text { wave-number components } \\ \delta & =\text { boundary-layer thickness } \\ \phi & =\text { porosity } \\ \mu & =\text { viscosity } \\ \lambda & =\text { disturbance wavelength }\end{array}$

\footnotetext{
* Associate Professor, Dept. of Aeromechanics and Flight Engineering, Senior Member AIAA

${ }^{\dagger}$ Principal Aeroacoustic Engineer, current address: Exa Corporation, Brisbane, CA 94005; Member AIAA

¥ Graduate student, Dept. of Mechanical Engineering

${ }^{\S}$ Professor, Dept. of Mechanical Engineering; Member AIAA
} 
$\theta$

$\omega$

Superscript

Subscripts

e

$w$
$=$ amplitude of temperature disturbance

$=$ complex circular frequency

= dimensional quantity

$=$ upper boundary-layer edge

$=$ quantity at the wall

\section{Introduction}

$\mathrm{L}$ aminar-turbulent transition in high-speed boundary layer flow leads to a significant increase in heat transfer and $\boldsymbol{L}_{\text {viscous drag. }}{ }^{1-3}$ This motivates the development of laminar flow control (LFC) methods aimed at increasing the laminar run. ${ }^{4}$ For predominately 2D configurations with sufficiently sharp leading edges (e.g. the X-43A vehicle) the dominant instability is associated with the Mack second mode and, presumably, the transition onset can be substantially delayed by stabilization of this mode. Fedorov et al. ${ }^{5}$ showed theoretically that a thin porous coating can stabilize the second mode by extracting a portion of disturbance energy. The experimental and theoretical studies $^{6-14}$ demonstrated the robustness of this LFC concept. It has been shown that porous coatings of random and regular microstructures can suppress the second-mode growth and significantly delay the transition onset on a sharp cone at zero angle of attack.

To address issues of flow non-uniformities associated with the boundary layer growth, separation and junctures between coated and uncoated regions, two-dimensional (2D) direct numerical simulations (DNS) have been carried out for near-wall flows over a flat plate, sharp cone and compression corner at free-stream Mach numbers 5-6. ${ }^{15}$ The impedance boundary conditions on the UAC surface were formulated using the theoretical model. ${ }^{5,7,8}$ It was shown that the coating end effects associated with the upstream and downstream boundaries of the coated region are local and can be neglected in calculations of the UAC integral performance. For the compression corner flow, the porous coating weakly affects disturbances in the separation region and strongly stabilizes the second-mode waves in the reattached boundary layer. Using a similar approach receptivity to freestream acoustic waves radiating a flat plate with UAC has been numerically simulated for the freestream Mach number $6 .{ }^{16}$ It was found that that a porous coating of regular porosity (equally spaced cylindrical blind micro-holes) effectively diminishes the second-mode growth rate, while weakly affecting acoustic waves.

The theoretical model ${ }^{5,7,8}$ of the impedance boundary conditions on the porous wall surface has several limiting assumptions. First, it is assumed that the UAC roughness is negligibly small; i.e., micro-cavities do not trip the boundary layer. The theory does not provide specific restrictions on the pore size. Second, because interactions between neighboring cavities are neglected, there are no restrictions of the pore spacing and/or porosity. Third, acoustic characteristics of isolated pores are evaluated using solutions valid far from the pore mouth and bottom. They are extrapolated to the pore opening by neglecting the end effects. To validate these assumptions Brès et al. ${ }^{17}$ have carried out 2D DNS of the interaction of incident acoustic waves with an array of equally spaced microcavities on a flat plate surface without flow. The reflection coefficient, characterizing the ratio of the reflected wave amplitude to the incident wave amplitude, has been computed as a function of the acoustic wave frequency and angle of incidence, for coatings of different porosity, at various acoustic Reynolds numbers. Overall, the numerical results confirm the theoretical predictions. The simulations also highlighted the presence of resonant acoustic modes caused by coupling of small-scale scattered waves near the porous surface. The cavity aspect ratio and the porosity were identified as the most important parameters. Guidelines for the choice of these parameters were suggested.

In Refs. 18 and 19, DNS of 2D disturbances has been conducted for the temporally evolving boundary layer on the aforementioned porous surface. Numerical solutions were obtained for relatively deep pores operating in attenuative regimes as well as for shallow pores operating in cancellation/reinforcement regimes with alternating regions of local minima and maxima of the acoustic absorption. In most cases, the numerical simulations agree with the linear instability theory that employs impedance boundary conditions.

The theoretical predictions were also compared with the numerical simulations of Sandham and Lüdeke ${ }^{20}$ for the boundary layer on a thermally insulated wall at freestream Mach $6 .{ }^{21}$ It was shown that for the coatings comprising equally spaced spanwise grooves and pores of rectangular cross-sections, the theory agrees well with the numerical solutions. Similar comparisons have been made by Wartemann and Lüdeke. ${ }^{22}$ Good agreement for different porous wall cases with adiabatic as well as cold wall condition was demonstrated. It turned out that the theoretical model holds in a much wider range of the UAC thickness and pore sizes than it was expected before. It is feasible to relax the originally conservative restrictions on the UAC parameters and seek optimal porous coatings in a wider 
parameter space. ${ }^{21}$ For instance, it is feasible to reduce the porous-layer thickness and thereby facilitate the UAC manufacturing with increased UAC effectiveness. This feasibility has been confirmed experimentally. ${ }^{23}$ Stability measurements on the UAC of various thicknesses showed that, in accord with theoretical and DNS predictions, an optimal UAC is approximately five times thinner than coatings tested in previous experiments.

Another important parameter, which strongly affects the UAC performance, is porosity. Because the UAC admittance increases with porosity, it is natural to assume that an optimal coating should have closely spaced pores. However, the numerical simulations ${ }^{19}$ revealed a new unstable mode on the UAC of high porosity. It was found that the coating having $2 \mathrm{D}$ cavities of aspect ratio 0.3 and porosity 0.8 triggers the new instability whose growth rate can be larger than that of Mack second mode. The LFC performance of this and similar coatings of high porosity may be reduced significantly. In this paper we investigate the nature of the new instability and identify the coating parameters at which this detrimental effect may occur.

\section{Numerical simulations}

\section{A. Problem formulation and numerical setup}

Consider 2D boundary-layer flow on a flat plate covered by a porous coating comprising equally spaced 2D cavities (figure 1). Each cavity has the depth $H^{*}$, half-width $b^{*}$ and spacing $s^{*}$. Hereafter asterisks denote dimensional quantities. The porous coating is characterized by the cavity aspect ratio $A r=2 b^{*} / H^{*}$ and porosity $\phi=2 b^{*} / s^{*}$. In our previous works, ${ }^{18,19}$ a simplified configuration that considers a $2 \mathrm{D}$ temporally evolving boundary layer on an infinite flat plate with this type of coating was shown to accurately capture the relevant flow physics.

(a)

(b)

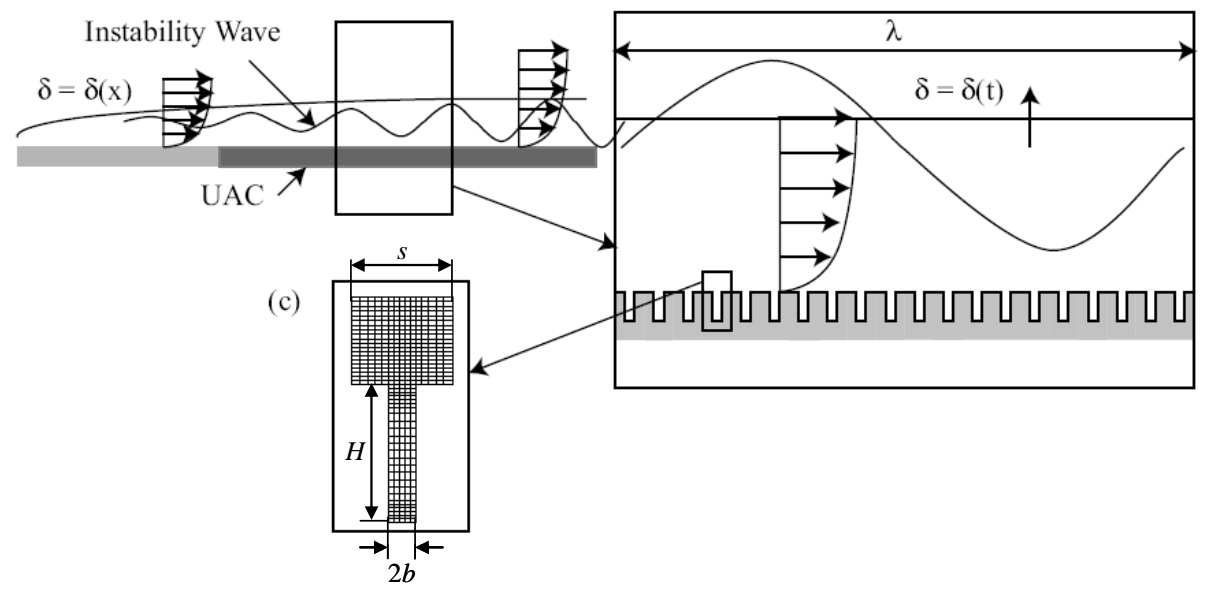

Figure 1. Schematic diagram for DNS of UAC effects: (a) the spatial problem with many wavelengths of the spatially growing instability wave; (b) the temporal problem with a single wavelength of the temporally growing instability wave; (c) a portion of the computational grid around a single pore.

The temporally evolving boundary layer neglects the spatial growth of the boundary layer, and instead diffuses slowly with time. Over short time-scales associated with acoustic energy attenuation in the coating, the laminar boundary layer is essentially frozen, consistent with either a spatial or temporal description of the mean flow field. Details on the code algorithm, implementation and validations can be found in Refs. 18, 24 and 25.

In the current study, simulations are performed for linearized (LDNS) two-dimensional Navier-Stokes equations. Periodic boundary conditions are used in the streamwise direction, and the nominally laminar boundary layer spreads in time rather than streamwise position. The grid extends up to $5 \mathrm{H}$ in the y-direction, with a large buffer zone at the top boundary, to avoid spurious reflections. The streamwise extent of the domain was chosen to approximately correspond to the wavelength of the most unstable mode, and to have an integer number of cavities in the domain. All the numerical simulations are performed on similar stretched Cartesian grids, with clustering of points near the walls. The mesh contains about half a million grid points, with 100 points per cavity depth, and 12 to 60 points across each cavity length, depending on the pore aspect ratio. 
The equations are solved for a perfect gas, with constant specific heat ratio $\gamma=1.4$ and Prandtl number $\operatorname{Pr}=$ 0.7. Here, the Mach number is $M_{e}=U_{e} / a_{e}=6$, and the wall temperature ratio is $T_{w}=T_{w}^{*} / T_{e}^{*}=1.4$. The viscosity coefficient was chosen to be constant $\left(\mu=\mu_{e}\right)$ or to be proportional to the gas temperature $\left(\mu^{*} / \mu_{e}^{*}=T^{*} / T_{e}^{*}\right)$.

Computations are initialized with an error-function profile for the streamwise velocity (i.e., the correct selfsimilar solution as $M_{e} \rightarrow 0$ ), uniform pressure and use the Crocco-Busemann relation to compute the initial temperature profile for the chosen wall temperature ratio. The nonlinear simulations are advanced in time until the boundary layer thickness is $\delta=2 H^{*}$. The resulting boundary layer profile is then frozen and used as the mean flow in linear simulations. An acoustic perturbation is added to the flow and the linearized Navier-Stokes equations are solved. The least damped (or most unstable) eigenmode is then determined from the long-time linear response of the boundary layer, and the nondimensionalized wavelength $\lambda=\lambda^{*} / \delta$, temporal growth/damping rate $\omega_{i}=\omega_{i}^{*} \delta / U_{e}$ and circular frequency $\omega_{r}=\omega_{i}^{*} \delta / U_{e}$ are computed. For analysis we also use the wavenumber $\alpha=2 \pi / \lambda$ and the complex phase speed $c=c_{r}+i c_{i}$, where $c_{r}=\omega_{r} / \alpha$ and $c_{i}=\omega_{i} / \alpha$.

\section{B. LDNS results}

The numerical simulations have been conducted for coatings of high porosity $\phi=0.8$. The Reynolds number $\operatorname{Re} \equiv H^{*} \rho_{e}^{*} U_{e}^{*} / \mu_{e}^{*}=12000$ corresponds to the boundary-layer thickness $\delta=2.003 H^{*}$ and the displacement thickness $\delta^{*}=1.1711 H^{*}$. The parameters of different cases and characteristics of the dominant mode predicted by LDNS are given in Table 1.

\begin{tabular}{|l|l|l|l|l|l|l|l|l|}
\hline Case & $A r$ & Viscosity law & \multicolumn{1}{|c|}{$\lambda$} & \multicolumn{1}{|c|}{$\alpha$} & \multicolumn{1}{|c|}{$\omega_{r}$} & $\omega_{i}$ & $c_{r}$ & \multicolumn{1}{c|}{$c_{i}$} \\
\hline 1 & 0.3 & $\mu=$ const & 1.219 & 5.1554 & 0.739 & 0.03833 & 0.143 & 0.00743 \\
\cline { 4 - 9 } & & & 2.4375 & 2.5777 & 0.7107 & 0.035 & 0.276 & 0.0136 \\
\hline 2 & 0.12 & $\mu \sim T$ & 2.470 & 2.544 & 0.614 & -0.0005 & 0.241 & -0.0002 \\
\hline 3 & 0.3 & $\mu \sim T$ & 2.433 & 2.582 & 0.669 & 0.0465 & 0.259 & 0.018 \\
\hline
\end{tabular}

Table 1. Parameters for the numerical simulations of a temporally-evolving boundary layer over a flat plate, the Reynolds number $\operatorname{Re}=12000$, porosity $\phi=0.8$.

For all the cases, parameters of this mode do not correspond to the Mack second mode, nor the acoustic resonant mode discussed in Ref. 19. This is a slow supersonic mode with the phase speed being essentially smaller than $1-1 / M_{e} \approx 0.833$. Because its eigenfrequency is close to the continuous spectrum branch of slow acoustic waves, its eigenfunction should oscillate outside the boundary layer. This is confirmed by snapshots of the disturbance pressure field shown in figure 2 for cases 2 and 3.
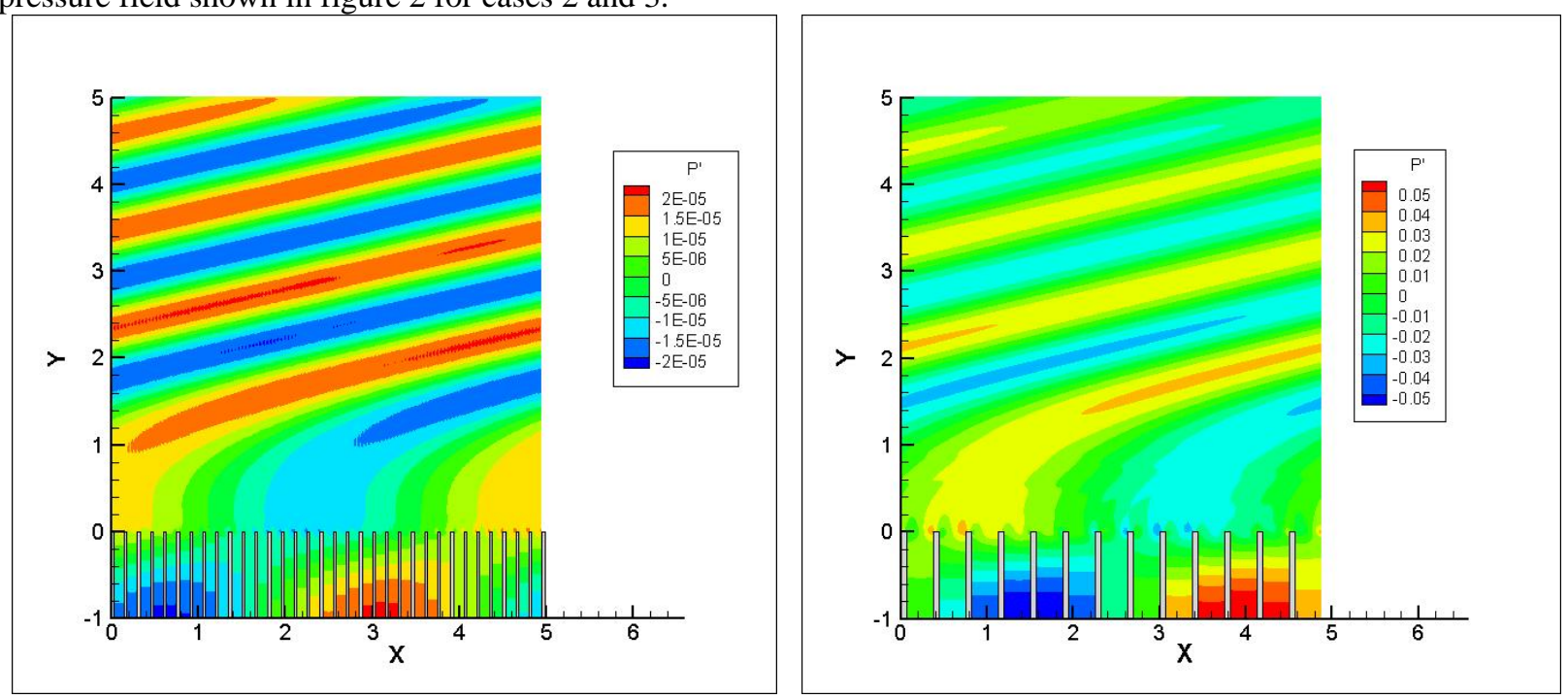

Figure 2. Snapshots of the pressure disturbance fields for case 2 (left panel) and case 3 (right panel). 


\section{LST analysis}

\section{A. Problem formulation}

The linear stability problem is solved using the DNS mean-flow solution on the solid (uncoated) wall. 3D disturbances are represented in the traveling-wave form

$$
q=\operatorname{Real}\{\hat{q}(y) \exp [i(\alpha x+\beta z-\omega t)]\}, \quad q=(u, v, w, p, \theta),
$$

where $(u, v, w)=\left(u^{*}, v^{*}, w^{*}\right) / U_{e}^{*}$ are velocity components; $p=p^{*} / \rho_{e}^{*} U_{e}^{* 2}$ is pressure, and $\theta=\theta^{*} / T_{e}^{*}$ is temperature. The nondimensional coordinates and time are $(x, y, z)=\left(x^{*}, y^{*}, z^{*}\right) / \delta$ and $t=t^{*} U_{e}^{*} / \delta$, respectively. For the parallel boundary-layer flow, the system of stability equations is solved with the boundary conditions on the porous surface

$$
\hat{u}(0)=0, \hat{v}(0)=A \hat{p}(0), \hat{w}(0)=0, \hat{\theta}(0)=0,
$$

where the porous-layer admittance $A$ is calculated as as,7, $^{5,8}$

$$
A=-\frac{\phi}{Z_{0}} \tanh (m H) .
$$

The characteristic impedance $Z_{0}$ and the propagation constant $m$ are expressed in terms of the complex dynamic density $\tilde{\rho}=\tilde{\rho}^{*} / \rho_{w}^{*}$ and complex compressibility $\tilde{C}=\gamma p_{w}^{*} \tilde{C}^{*}$ as

$$
Z_{0}=\frac{\sqrt{\tilde{\rho} / \tilde{C}}}{M_{e} \sqrt{T_{w}}}, m=\frac{i \omega M_{e}}{\sqrt{T_{w}}} \sqrt{\tilde{\rho} \tilde{C}} .
$$

The quantities of $\tilde{\rho}$ and $\tilde{C}$ are calculated using the analytical solutions ${ }^{9}$ of the problem describing propagation of harmonic disturbances within a deep cavity

$$
\begin{gathered}
\tilde{\rho}=1 /[1-F(\xi)], \tilde{C}=1+(\gamma-1) F(\tilde{\xi}), \\
F(\xi)=\tan \xi / \xi,
\end{gathered}
$$

where $\xi=\sqrt{i \omega^{*} \rho_{w}^{*} b^{* 2} / \mu_{w}^{*}}$ characterizes the ratio of the cavity half-width $b^{*}$ to the Stokes layer thickness $\sqrt{\mu_{w}^{*} / \rho_{w}^{*} \omega^{*}}$, and $\tilde{\xi}=\xi \sqrt{\operatorname{Pr}}$. In the solid-wall case, $A=0$, the boundary conditions (2) have a standard form $u(0)=v(0)=w(0)=\theta(0)=0$.

Outside the boundary layer, $y \rightarrow \infty$, the eigenmodes decay exponentially

$$
\hat{u}(\infty)=\hat{v}(\infty)=\hat{w}(\infty)=\hat{\theta}(\infty)=0 .
$$

The stability equations are integrated from the outer boundary-layer edge to the wall using a $4^{\text {th }}$-order RungeKutta scheme and a Gram-Schmidt orthonormalization procedure. From known boundary conditions in the freestream, the eigenvalues are found using a shooting/Newton-Raphson procedure in order to satisfy the wall boundary conditions (2). At convergence, the eigenvalues are related through the numerically obtained dispersion relation $D(\alpha, \beta, \omega)=0$. For the temporal stability problem, the wavenumbers $\alpha$ and $\beta$ are real, whereas $\omega$ is complex eigenfrequency.

Note that the boundary conditions on the porous wall were obtained in the following framework: 1) slip effects leading to perturbations of $u(0)$ and $w(0)$ are ignored; 2) the temperature perturbation $\theta(0)$ is neglected; 3$)$ the cross-sectional pore size is small compared with the pore length; 4) the interaction between disturbances in the neighboring pores is neglected; 5) the number of pores per disturbance wavelength is large; 6) the coating roughness is negligible; 7) the mean temperature of the porous layer is uniform. 


\section{B. Results}

Hereafter the boundary-layer mean flow corresponds to the Reynolds number $\operatorname{Re}_{\delta}=\operatorname{Re} \cdot \delta / H^{*}=24000$ on the solid wall. We start with the LST analysis for the solid-wall case at viscosity $\mu \sim T$. Figure 3 shows that the spectrum behavior $\omega(\alpha)$ is typical for a hypersonic boundary layer. ${ }^{26,27}$ Namely, for small wavenumbers $\alpha$, the fast mode (solid line) coalesces with fast acoustic waves of the phase speed $c=1+1 / M$ and the slow mode (dashed line) coalesces with slow acoustic waves of $c=1-1 / M$. Owing to synchronization of these modes in the vicinity of $\alpha \approx 2.4$, branching of the dispersion curves leads to destabilization of the fast mode and stabilization of the slow mode. Here the unstable fast mode corresponds to the Mack second mode.

As shown in figure 4, the second-mode instability is effectively suppressed by the porous coatings having relatively deep cavities. For small porosity $\phi \leq 0.1$ (black and red lines), the second-mode instability corresponds to the fast mode having $c_{r} \rightarrow 1+1 / M$ as $\alpha \rightarrow 0$. For higher porosity, the instability corresponds to the slow mode having $c_{r} \rightarrow 1-1 / M$ as $\alpha \rightarrow 0$. Such switching over indicates that the spectrum branch point moves from one half-plane of complex $\alpha$ to the other as porosity increases. ${ }^{27}$

Similar behavior of the disturbance spectrum is observed in the case of $\mu=$ const. As shown in figure 5, the second-mode instability is associated with the fast mode for $\phi \leq 0.4$ and with the slow mode for higher porosity. The porous coating effectively suppresses this instability.
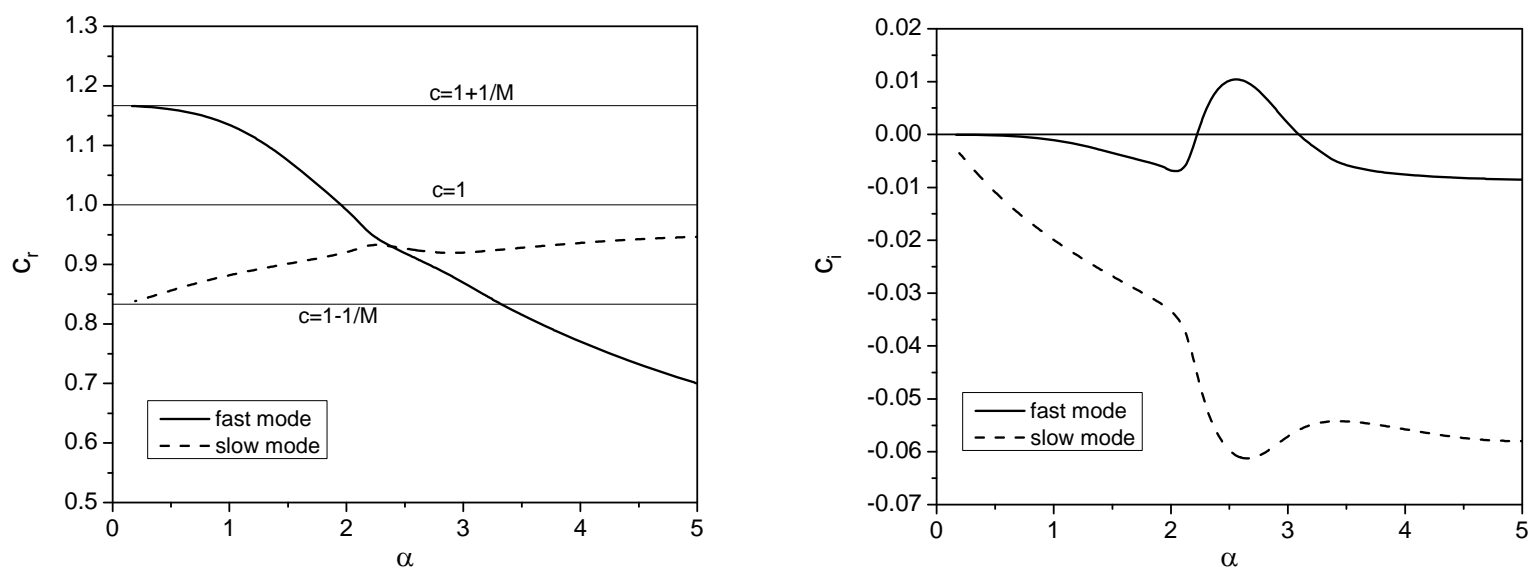

Figure 3. Disturbance spectrum $c(\alpha)$ for the solid wall case, $\operatorname{Re}_{\delta}=24000, \mu \sim T$.
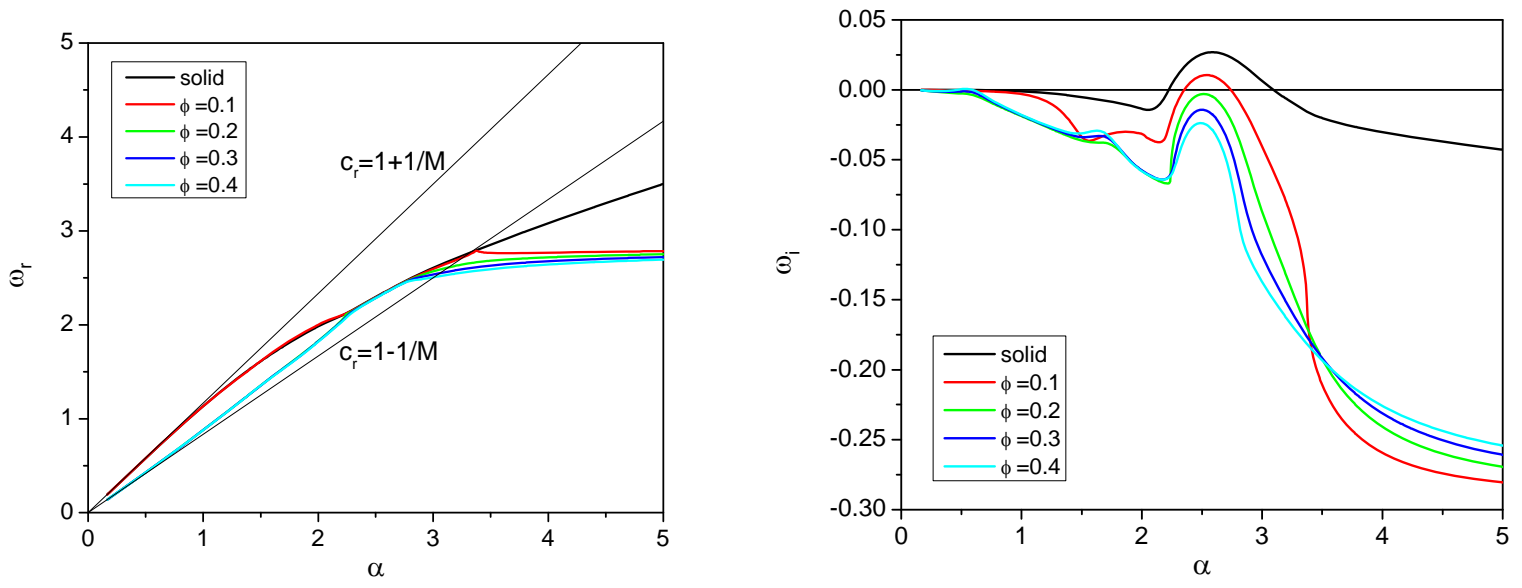

Figure 4. Stabilization of Mack second mode by porous coatings having cavities of the aspect ratio $A r=0.12$,

$$
\operatorname{Re}_{\delta}=24000, \mu \sim T \text {. }
$$



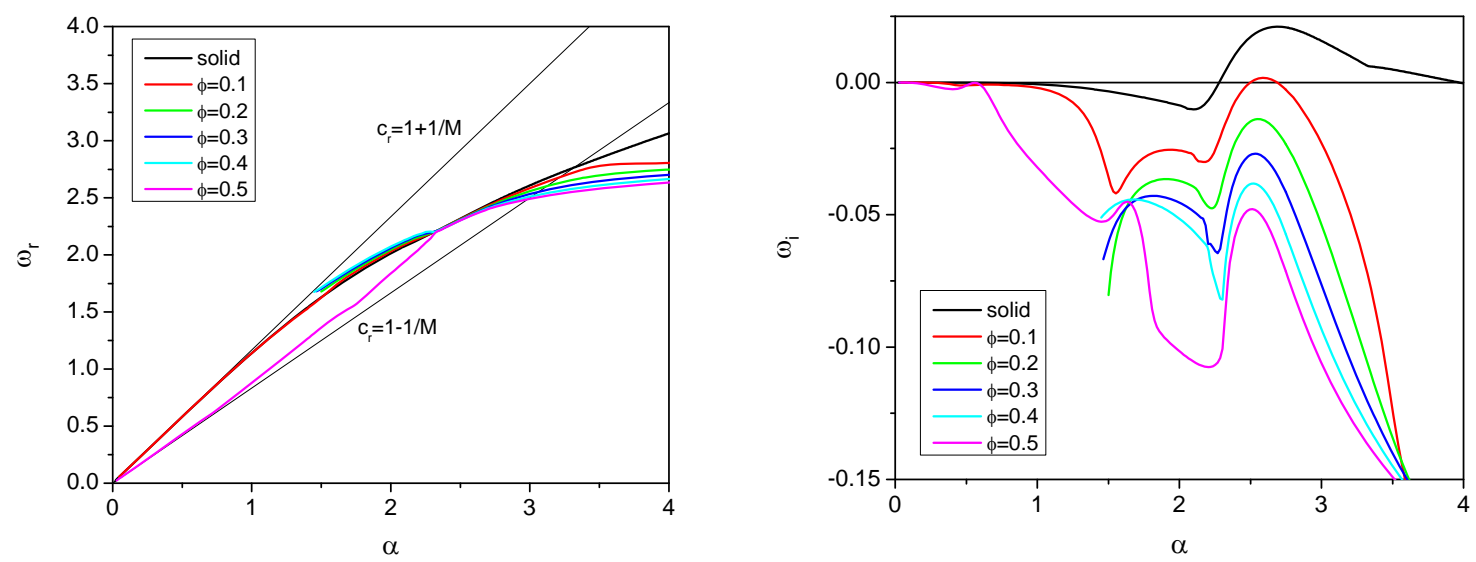

Figure 5. Stabilization of Mack second mode by porous coatings having cavities of the aspect ratio $A r=0.12$, $\operatorname{Re}_{\delta}=24000, \mu=$ const .

The situation changes dramatically in the case of high porosity and relatively shallow cavities. As an example, figure 6 shows the disturbance spectrum for the coating of $\phi=0.8$ and $A r=0.3$ (case 1 in Table 1). Besides fast and slow modes (red and blue lines), which are typical for the solid-wall and moderate porosity cases, there is a new unstable mode (black line). Figure 7 shows that the new instability occurs in a wide range of the wavelength $\lambda$ and has significant growth rates, which can be larger than the second-mode growth rate in the solid-wall case. In the unstable region, the phase speed is rather small. The physical mechanism causing this deceleration is discussed below. The phase speed trajectory of the new mode in the complex $c$-plane is shown in figure 8. As $\alpha$ increases, the phase speed departs from the branch-cut of slow acoustic waves, enters to the upper half-plane related to unstable waves and moves over this branch-cut in its close vicinity. The LDNS data shown in figures 6-8 by circles agree with the theory. Figure 9 shows that the pressure disturbance fields predicted by LST (black lines) and LDNS (colors) are very close. Outside the boundary layer, the disturbance behaves as an outgoing acoustic wave with the wave front having small angle with respect to the wall.

It is natural to assume that the new instability is associated with resonant interactions in the cavities. Indeed, figures 6 and 7 show that the frequency and phase speed of the new mode are close to the first acoustic resonance determined by the condition $H^{*} \omega^{*} / a_{w}^{*}=\pi / 2$ for an isolated cavity of small aspect ratio. In this case, each cavity works as an acoustic resonator that is effectively excited at the lowest frequency corresponding to $H^{*}=\lambda_{w}^{*} / 4$, where $\lambda_{w}^{*}=a_{w}^{*} / f^{*}$ is acoustic wavelength.
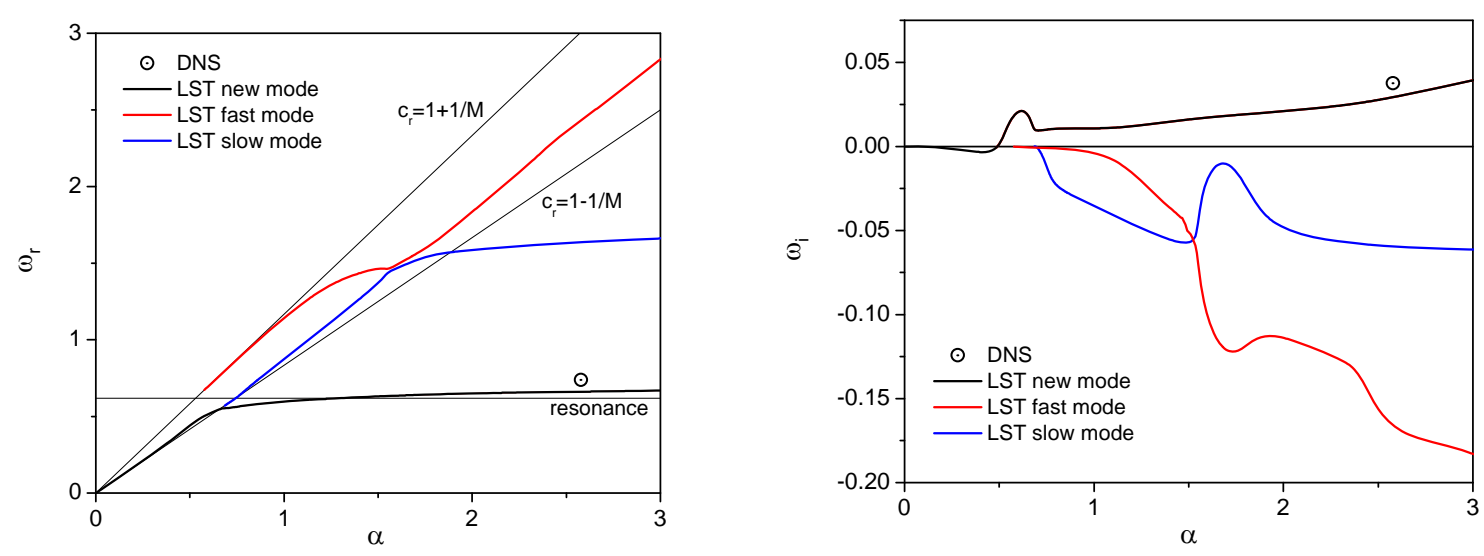

Figure 6. Disturbance spectrum for the porous wall of $A r=0.3$ and $\phi=0.8, \operatorname{Re}_{\delta}=24000, \mu=$ const . 

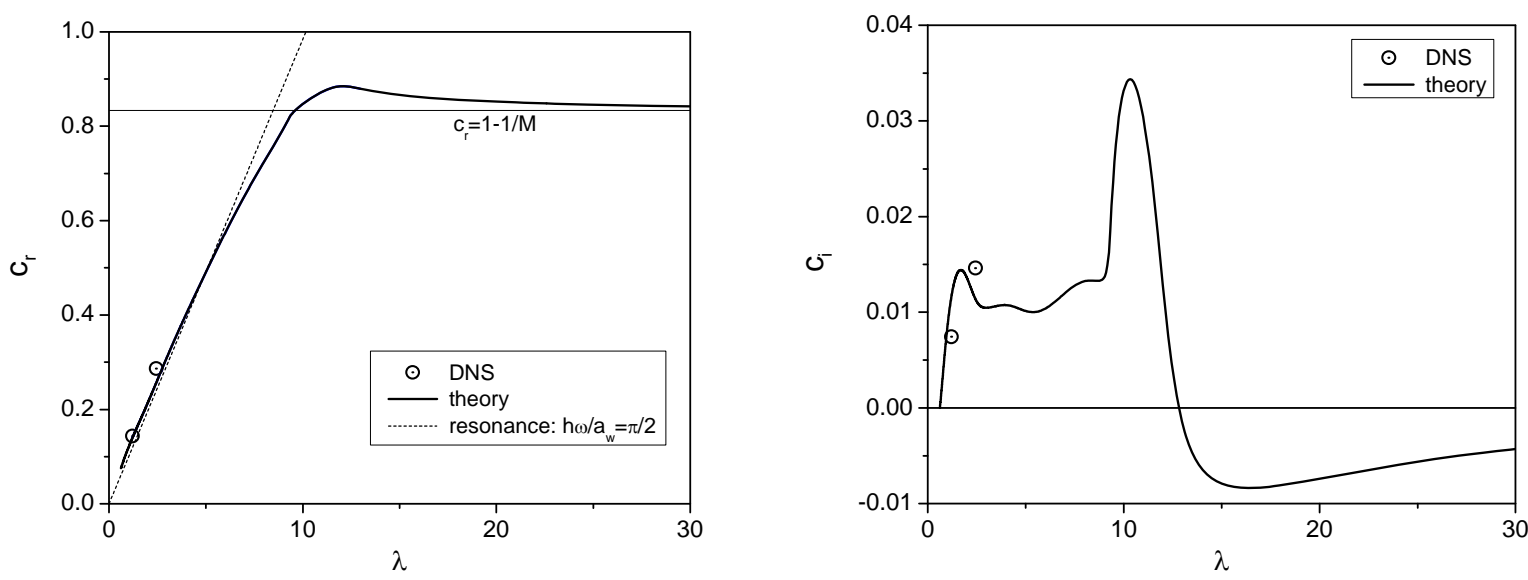

Figure 7. The phase speed of new unstable mode versus the wavelength $\lambda . A r=0.3, \phi=0.8, \operatorname{Re}_{\delta}=24000$, $\mu=$ const .

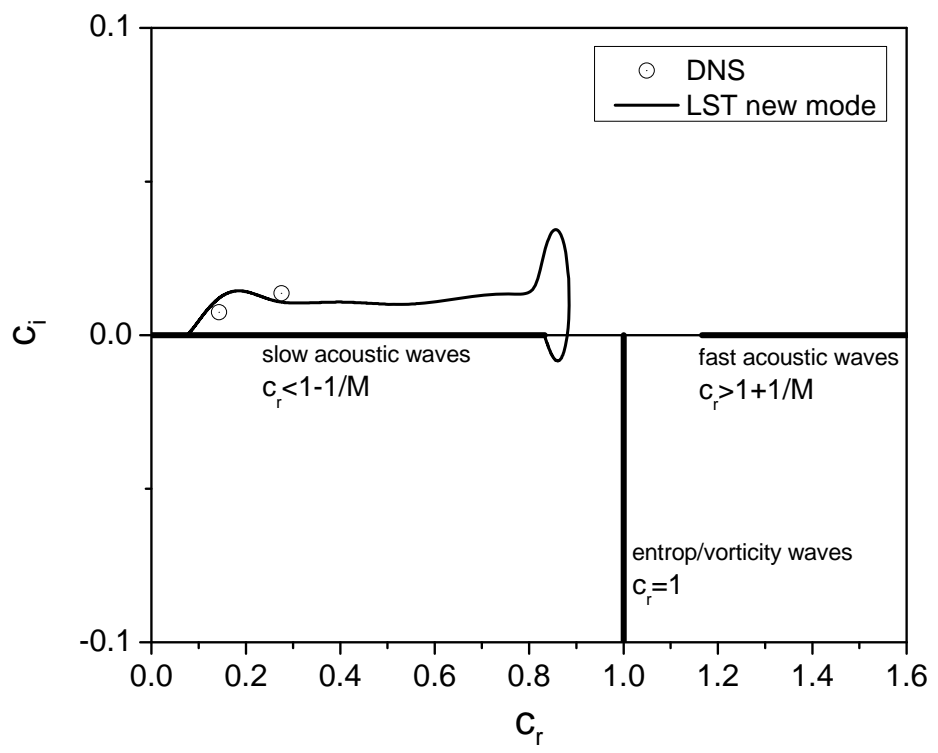

Figure 8. The phase-speed trajectory of the new mode in the complex $c$-plane. $A r=0.3, \phi=0.8, \operatorname{Re}_{\delta}=24000$, $\mu=$ const .

To shed light on the physical mechanism causing deceleration of the disturbance over a porous surface, we consider a simple case - a 2D inviscid wave propagating over a grooved wall without external flow (figure 10). Over the wall, the pressure disturbance $p=\hat{p}(x, y) \exp (-i \omega t)$ is governed by the Helmholtz equation

$$
\Delta \hat{p}+\left(\frac{\omega}{a_{w}}\right)^{2} \hat{p}=0
$$

which has the general solution

$$
\begin{array}{r}
\hat{p}(x, y)=\hat{p}_{+} \exp (i \alpha x+\kappa y)+\hat{p}_{-} \exp (i \alpha x-\kappa y), \\
\kappa^{2}=\alpha^{2}-\left(\frac{\omega}{a_{w}}\right)^{2}, \text { Real } \kappa>0 .
\end{array}
$$




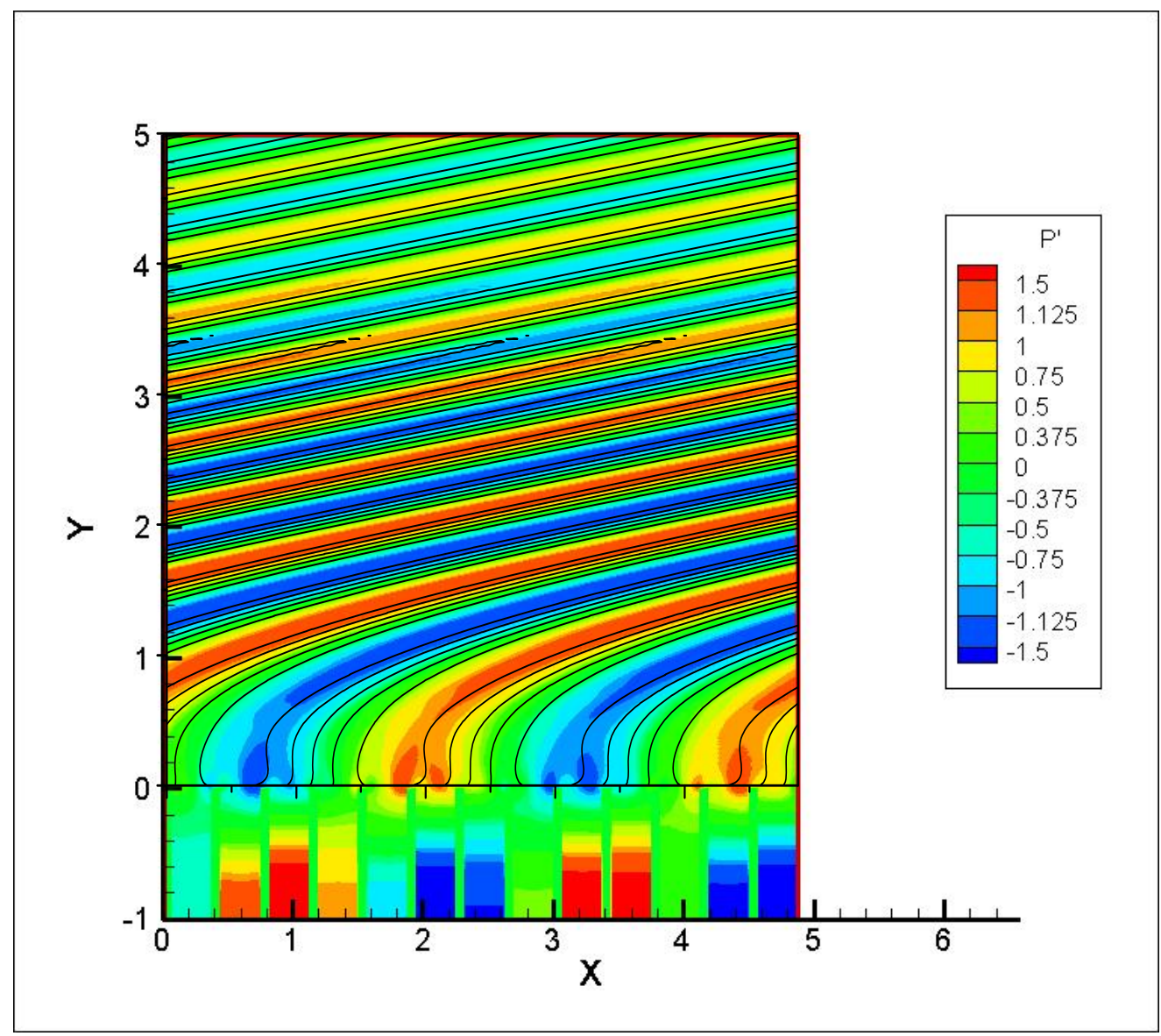

Figure 9. Pressure disturbance field predicted by LDNS (color) and LST (black lines), wavelength $\lambda=1.219$, case 1, here $X=x^{*} / H^{*}$ and $Y=y^{*} / H^{*}$.

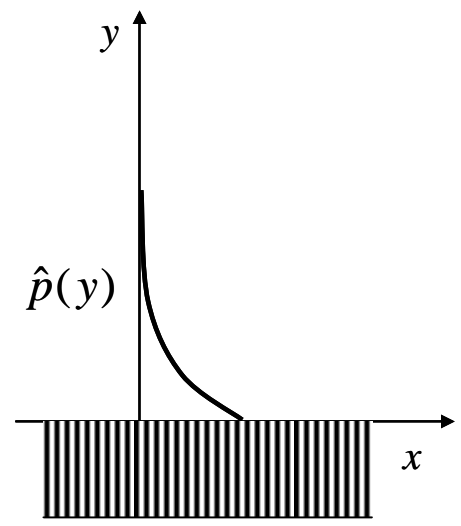

Figure 10. Near-wall wave propagating over a grooved layer comprising closely spaced deep cavities.

For a wave propagating with the phase speed $c<a_{w}$, we have $\kappa=\alpha \sqrt{1-\frac{c^{2}}{a_{w}^{2}}}$, where $\alpha>0$. Since the near-wall wave decays as $y \rightarrow \infty$, we get

$$
\hat{p}(x, y)=\hat{p}_{-} \exp (i \alpha x-\kappa y) .
$$


The linearized $y$-momentum equation

$$
\frac{\partial v}{\partial t}+\frac{1}{\rho_{w}} \frac{\partial p}{\partial y}=0
$$

gives

$$
\begin{aligned}
& -i \omega \hat{v}_{-}-\frac{\kappa}{\rho_{w}} \hat{p}_{-}=0, \\
& \omega=\frac{i \kappa}{\rho_{w}} Z(\omega),
\end{aligned}
$$

where $Z(\omega)=\hat{p}_{-} / \hat{v}_{-}$is the porous wall impedance. Neglecting viscous effects inside cavities, we get

$$
Z=-i \frac{\rho_{w} a_{w}}{\phi} \cot \left(\omega H / a_{w}\right) .
$$

Substituting (13) and the expression for $\kappa$ into the dispersion relation (12), we obtain the wave phase speed

$$
\frac{c}{a_{w}}=\frac{1}{\sqrt{1+\phi^{2} \tan ^{2}\left(\omega H / a_{w}\right)}} .
$$

Equation (14) shows that the grooved coating leads to a decrease of the disturbance phase speed. As $\omega H / a_{w}$ approaches the resonance condition $\omega H / a_{w}=\pi / 2$, the phase speed tends to zero. Thus, disturbance fields near the cavity mouths are coupled such that the near-surface wave can propagate much slower than acoustic waves in unbounded medium.

The LST results for 3D (oblique) waves related to the new unstable mode are shown in figure 11 for $\lambda=1.219$ and 2.4374 corresponding to case 1. Maximal instability is observed for $2 \mathrm{D}$ waves of $\beta=0$, that is typical for acoustic instabilities of high-speed boundary-layer flows.

Now we consider dependencies of the new instability on the coating parameters. As shown in figure 12, the mode is stabilized as porosity decreases. Presumably this is due to the fact that the vertical velocity amplitude averaged over the porous surface decreases proportionally to $\phi$. Figure 13 illustrates the cavity depth effect where the eigenfrequency $\omega$ is shown as a function of the cavity aspect ratio $A r$ at the wavelength $\lambda=1.219$, the acoustic Reynolds number $\operatorname{Re}_{a} \equiv \rho_{w}^{*} a_{w}^{*} b^{*} / \mu_{w}^{*}=254.5$ and porosity $\phi=0.8$. Very deep cavities $(A r \ll 1)$ and very shallow cavities ( $A r>0.8$ ) do not resonate, and the mode is stable. The maximum instability is observed for cavities of moderate aspect ratio $A r \approx 0.3$ favorable for the resonance mechanism. Finally, figure 14 shows that the unstable region is formed in the vicinity of the resonance point $H^{*}=H_{r e s}^{*}=\pi a_{w}^{*} / 2 \omega^{*}$. Namely, the instability maximum is observed at $H_{\text {res }}^{*} / H^{*} \approx 0.9$.
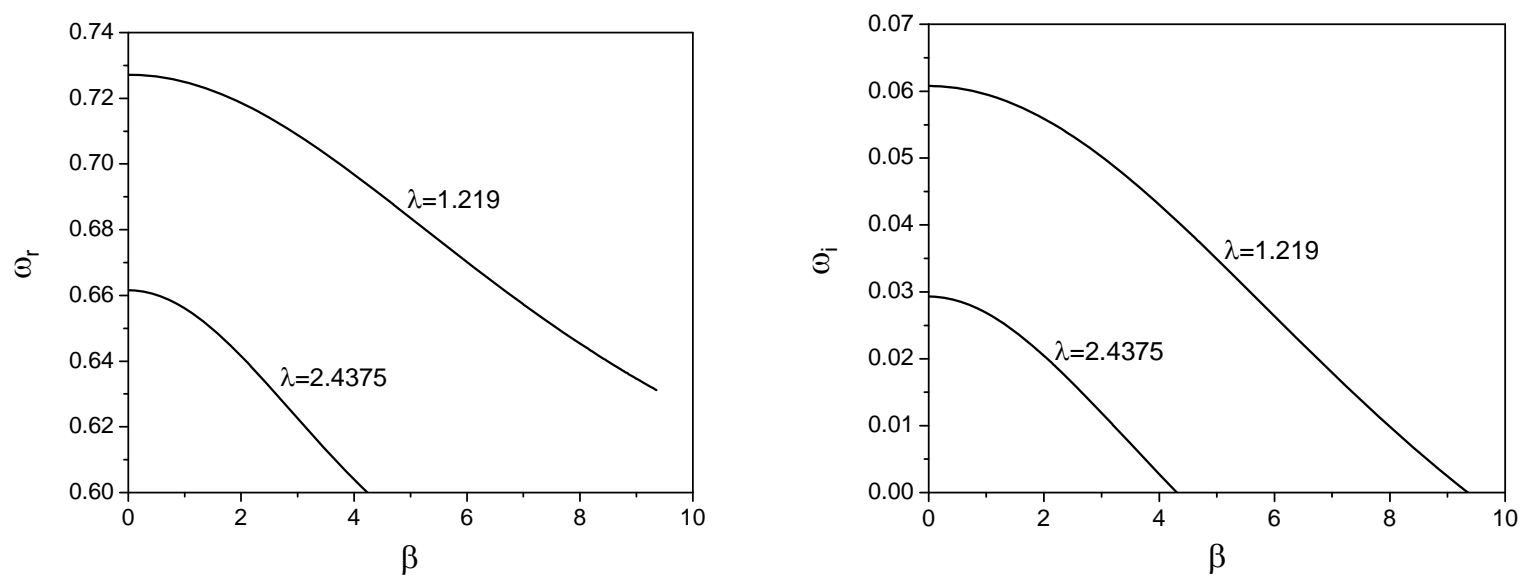

Figure 11. The distribution of $\omega(\beta)$ for the unstable mode. $A r=0.3, \phi=0.8, \operatorname{Re}_{\delta}=24000, \mu=$ const . 

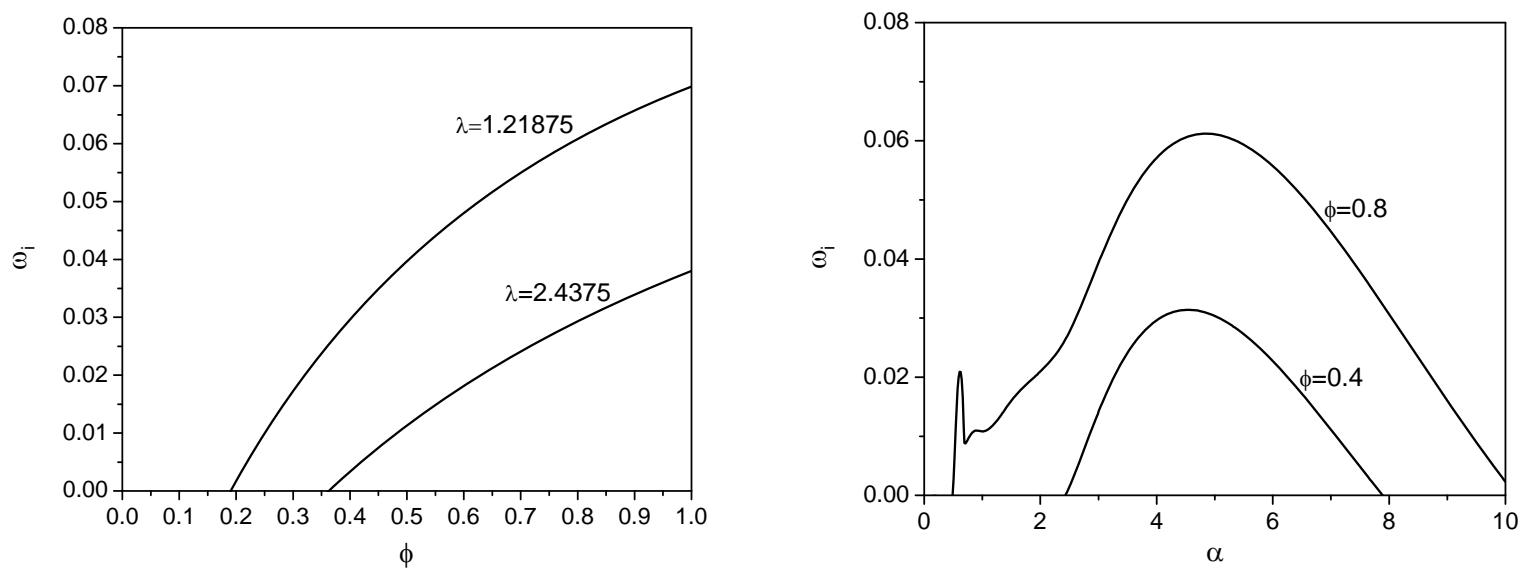

Figure 12. Distribution of $\omega_{i}(\phi)$ at fixed $\lambda$ (left panel) and $\omega_{i}(\alpha)$ at $\phi=0.4$ and 0.8 (right panel). $A r=0.3$, $\operatorname{Re}_{\delta}=24000, \mu=$ const .

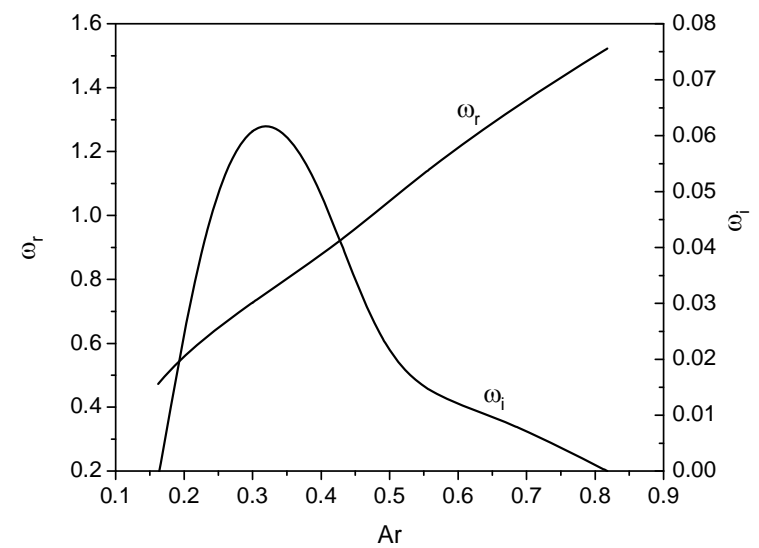

Figure 13. Distribution of $\omega(A r)$ at $\lambda=1.219$, $\operatorname{Re}_{a}=254.5$ and porosity $\phi=0.8, \mu=$ const.

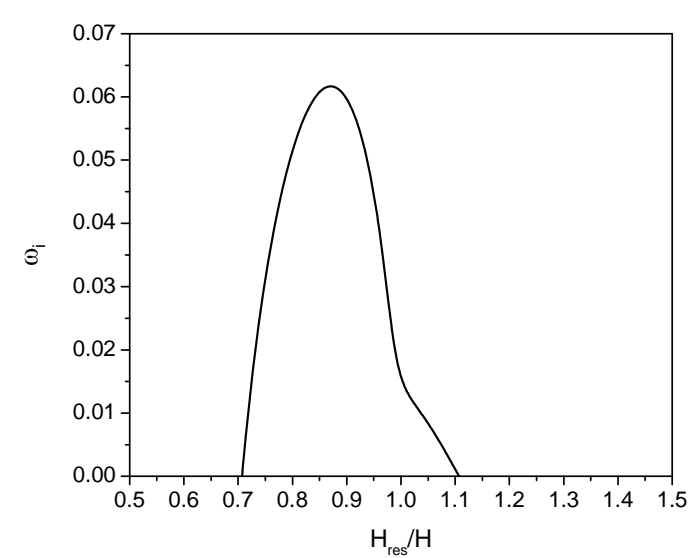

Fig. 14 The growth rate of slow mode vs. $H_{\text {res }}^{*} / H^{*}$. $\operatorname{Re}_{a}=254.5, \quad \phi=0.8, \quad \lambda=1.219, \mu=$ const.

The LST computations for case 3 ( $\mu \sim T$ ) give the phase speed of a new mode $c=0.2585+i 0.0269$. The pressure disturbance field predicted theoretically agrees very well with the LDNS solution (figure 15). The real part of $c$ is also close to the LDNS value given in Table 1, while the imaginary part is essentially larger. Presumably this discrepancy is caused by perturbations of the longitudinal velocity on the UAC surface. In the LST analysis, these perturbations are ignored and the boundary condition is formulated as $\hat{u}(0)=0$.

To check this assumption we consider the linearized equation for the $x$-momentum in the inviscid approximation

$$
\frac{1}{T}\left(\frac{\partial u}{\partial t}+U \frac{\partial u}{\partial x}+v \frac{\partial U}{\partial y}\right)=-\frac{\partial p}{\partial x} .
$$

The derivation of (15) takes into account that the mean flow has the longitudinal velocity $U=U(y)$, the vertical velocity $V=0$ and density $\rho=1 / T$. For disturbances of the wave form $q=\hat{q}(y) \exp (i \alpha x-i \omega t)$, Equation (15) reads

$$
\frac{1}{T}\left(-i \omega \hat{u}+i \alpha U \hat{u}+\hat{v} U^{\prime}\right)=-i \alpha \hat{p} .
$$

Near the wall ( $y \rightarrow 0$ ), the amplitude of longitudinal velocity is expressed as 


$$
\hat{u}(0)=\frac{-i U^{\prime}(0) \hat{v}(0)+\alpha T(0) \hat{p}(0)}{\omega}
$$

Equation (17) corresponds to pure slip. Because on the solid portions of the UAC surface $\hat{u}(0)=0$, the disturbance amplitude averaged over the porous surface is

$$
\hat{u}(0)=\phi \frac{-i U^{\prime}(0) \hat{v}(0)+\alpha T(0) \hat{p}(0)}{\omega}
$$

For first-cut estimates, we introduce a coefficient $K$ to account for the reduction of $\hat{u}(0)$ on the cavity mouth by viscous effects. Then the boundary condition for the $x$-velocity amplitude is approximated as

$$
\hat{u}(0)=K \phi \frac{-i U^{\prime}(0) \hat{v}(0)+\alpha T(0) \hat{p}(0)}{\omega}
$$

The LST computations show that the boundary condition (19) leads to significant reduction of the new mode growth rate $\omega_{i}$, while weakly affecting $\omega_{r}$. At $K=0.9$ the new-mode phase speed $c=0.2533+i 0.0181$ is close to the LDNS phase speed (case 3 from Table 1).

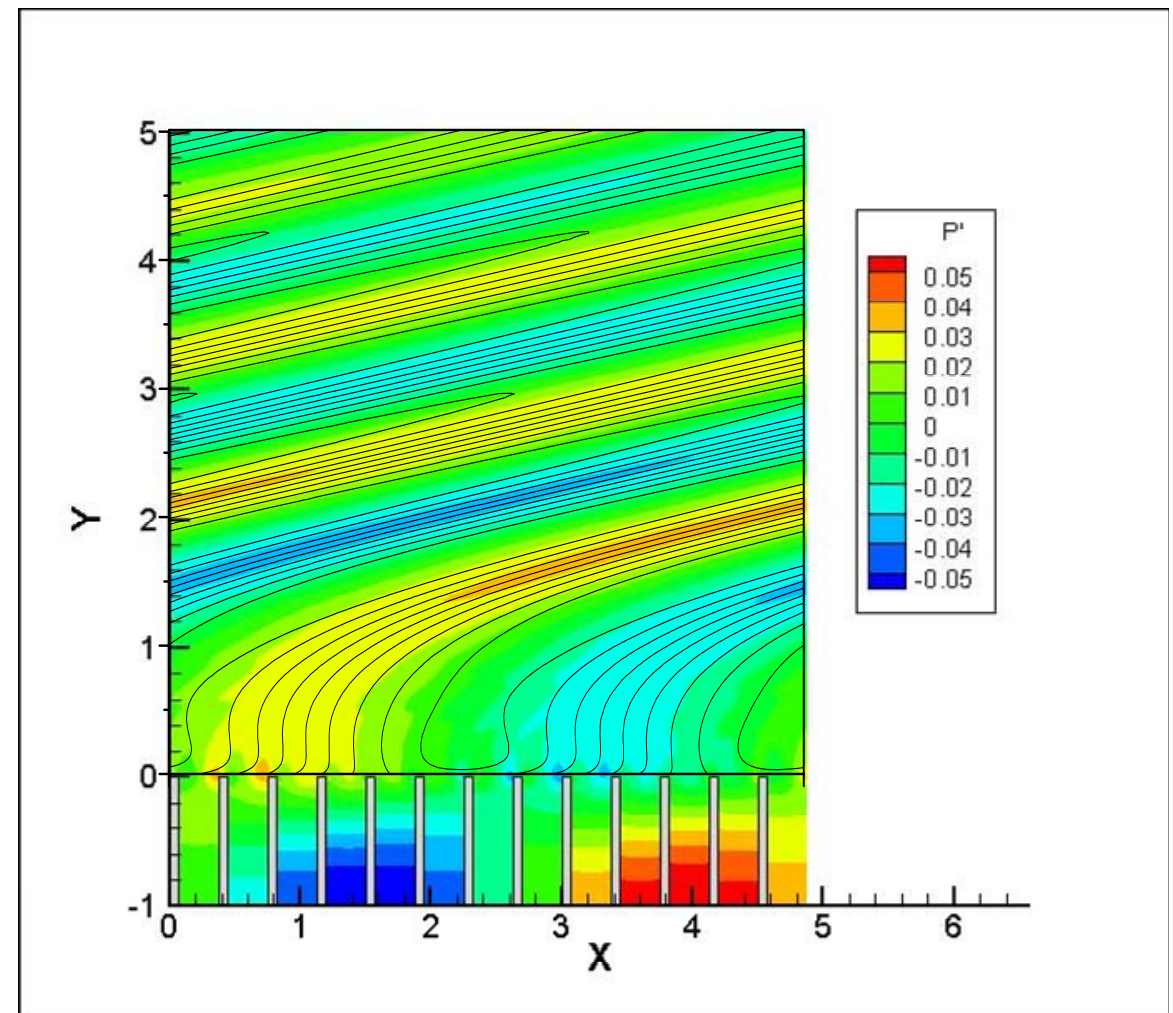

Figure 15. Pressure disturbance field predicted by LDNS (color) and LST (black lines), wavelength $\lambda=2.433$, case 3 from Table 1, here $X=x^{*} / H^{*}$ and $Y=y^{*} / H^{*}$.

\section{Summary}

2D LDNS and LST analyses have been performed for the temporally evolving boundary layer on a flat wall at the outer-flow Mach number 6 . The wall is covered by a porous coating consisting of equally spaced rectangular micro-cavities oriented in the spanwise direction.

For the coating with cavities of aspect ratio 0.3 and porosity 0.8 , a new unstable mode was identified numerically. This mode propagates downstream with low phase speed and has a growth rate which can be larger than that of the most unstable second mode. The new instability was also captured by the linear stability theory with the impedance boundary conditions on the porous surface. In the case of $\mu=$ const, the eigenfrequencies and 
eigenfunctions predicted by LST agree with the LDNS data. In the case of $\mu \sim T$, the LST model overpredicts the new-mode growth rate. First-cut estimates indicated that this discrepancy may be caused by non-zero fluctuations of the longitudinal velocity on the porous surface.

The theoretical analysis showed that the new mode is associated with acoustic resonances in the cavities. Disturbance fields near mouths of resonating cavities are coupled such that the boundary-layer disturbance is decelerated and becomes unstable.

Parametric studies indicate that the new instability occurs on coatings having closely spaced cavities of moderate aspect ratio. To avoid this detrimental effect, which can reduce the UAC LFC performance, the coating should have sufficiently small porosity and/or narrow pores of sufficiently small aspect ratio. Restrictions on these parameters can be estimated using the linear stability theory with the impedance boundary conditions.

Note that previous theoretical, numerical and experimental studies of the UAC stabilization effect have been focused on the low-porosity and/or narrow-pore configurations, which do not provide favorable conditions for the foregoing instability mechanism. However, optimal UAC, providing maximal damping of the Mack second mode, have high porosity and relatively shallow cavities. ${ }^{19,23}$ Seeking this optimum, we should check that the porous coating does not sustain the new instability.

\section{Acknowledgments}

This work is supported partially (Fedorov) by the AFOSR/NASA National Center for Hypersonic Research in Laminar-Turbulent Transition. Computer time was provided in part by the Department of Defense High Performance Computing centers.

\section{References}

${ }^{1}$ Malik, M.R., Zang, T.A., and Bushnell, D.M., “Boundary Layer Transition in Hypersonic Flows,” AIAA-90-5232, 1990.

${ }^{2}$ Schneider, S.P., "Flight Data for Boundary layer Transition at Hypersonic and Supersonic Speeds," J. Spacecraft and Rockets, Vol. 36, No. 1, 1999, pp. 8-20.

${ }^{3}$ Schneider, S.P., "Hypersonic Laminar-Turbulent Transition on Circular Cones and Scramjet Forebodies," Progress in Aerospace Sciences, Vol. 40, No. 1-2, Feb. 2004, pp. 1-50.

${ }^{4}$ Kimmel, R.L., “Aspects of Hypersonic Boundary-Layer Transition Control,” AIAA-2003-772, 6-9 Jan. 2003, Reno, NV.

${ }^{5}$ Fedorov, A.V., Malmuth, N.D., Rasheed, A., and Hornung, H.G., "Stabilization of Hypersonic Boundary Layers by Porous Coatings,” AIAA J., Vol. 39, No. 4, April 2001, pp. 605-610.

${ }^{6}$ Rasheed, A., Hornung, H.G., Fedorov, A.V., and Malmuth, N.D., "Experiments on Passive Hypervelocity Boundary Layer Control Using an Ultrasonically Absorptive Surface,” AIAA J., Vol. 40, No. 3, pp. 481-489.

${ }^{7}$ Fedorov, A., Shiplyuk, A., Maslov, A., Burov, E., and Malmuth, N., "Stabilization of a Hypersonic Boundary Layer Using an Ultrasonically Absorptive Coating,” J. Fluid Mech., Vol. 479, 2003, pp. 99-124.

${ }^{8}$ Fedorov A., Kozlov V., Shiplyuk A., Maslov A., Sidorenko A., Burov E., and Malmuth N., "Stability of Hypersonic Boundary Layer on Porous Wall with Regular Microstructure,” AIAA-2003-4147, June 2003.

${ }^{9}$ Kozlov, V.F., Fedorov, A.V. and Malmuth, N.D., "Acoustic Properties of Rarified Gases Inside Pores of Simple

Geometries,“ J. Acoust. Soc. Am., Vol. 117, No. 6, June 2005, pp. 3402-3412.

${ }^{10}$ Chokani, N., Bountin, D.A., Shiplyuk, A.N., Maslov, A.A., and "Nonlinear Aspects of Hypersonic Boundary Layer Stability on a Porous Surface," AIAA J., Vol. 43, No. 1, Jan. 2005, pp. 149-155.

${ }^{11}$ Fedorov,A., Shiplyuk, A., Maslov, A., Kozlov, V., Sidorenko, A., and Malmuth, N., "Hypersonic Laminar Flow Control Using a Porous Coating of Random Microstructure,” AIAA-2006-1112, Jan. 2006, Reno, NV.

${ }^{12}$ Fedorov A.V., Kozlov V.F., and Addison, R.C., "Reflection of Acoustic Disturbances from a Porous Coating of Regular Microstructure,” AIAA-2008-3902, 23-26 June 2008, Seattle, Washington.

${ }^{13}$ Maslov, A.A., Fedorov, A.V., Bountin, D.A., Shiplyuk, A.N., Sidorenko, A.A., and Malmuth, N.D., "Experimental Study of Transition in Hypersonic Boundary Layer on Ultrasonically Absorptive Coating with Random Porosity,” AIAA2008-587, 7-10 Jan. 2008, Reno, NV.

${ }^{14}$ Fedorov A.V., and Malmuth, N.D., "Parametric Studies of Hypersonic Laminar Flow Control Using a Porous Coating of Regular Microstructure,” AIAA-2008-588, 7-10 Jan. 2008, Reno, NV.

${ }^{15}$ Egorov, I.V., Fedorov, A.V., Novikov, A.V., and Soudakov, V.G., "Direct Numerical Simulation of Supersonic Boundary-Layer Stabilization by Porous Coatings,” AIAA-2007-948, 8-11 Jan. 2007, Reno, NV.

${ }^{16}$ Egorov, I.V., Fedorov, A.V., and Soudakov, V.G., Receptivity of a Hypersonic Boundary Layer over a Flat Plate with a Porous Coating,” J. Fluid Mech., Vol. 601, 2008, pp. 165-187. 
${ }^{17}$ Brès, G.A., Colonius, T., and Fedorov, A.V., “Acoustic Properties of Porous Coatings for Hypersonic BoundaryLayer Control,” AIAA J., Vol. 48, No. 2, 2010, pp. 267-274. (see also AIAA-2008-3903, 23-26 Jun. 2008, Seattle, Washington).

${ }^{18}$ Brès, G.A., Colonius, T., and Fedorov, A.V., "Stability of Temporally Evolving Supersonic Boundary Layers over Micro-Cavities for Ultrasonic Absorptive Coatings,” AIAA-2008-4337, 23-26 Jun. 2008, Seattle, Washington.

${ }^{19}$ Brès, G.A., Inkman, M, Colonius, T., and Fedorov, A.V., “Alternate Designs of Ultrasonic Absorptive Coatings for Hypersonic Boundary Layer Control,” AIAA-2009-4217, 22-25 June 2009, San Antonio, Texas.

${ }^{20}$ Sandham, N.D., and Lüdeke, H., "Numerical Study of Mach 6 Boundary-Layer Stabilization by Means of a Porous Surface,” AIAA J., Vol. 47, No. 9, 2009, pp. 2243-2252. (see also AIAA-2009-1288, 5-8 Jan. 2008, Orlando, FL).

${ }^{21}$ Fedorov, A.V., "Temporal Stability of Hypersonic Boundary Layer on Porous Wall: Comparison of Theory with DNS," AIAA-2010-1242, 4-7 Jan. 2010, Orlando, FL.

${ }^{22}$ Wartemann, V., and Lüdeke, H., "Investigation of Slip Boundary Conditions of Hypersonic Flow over Microporous Surfaces,” V European Conference on Computational Fluid Dynamics, ECCOMAS CFD 2010, J.C.F. Pereira and A. Sequeira (Eds), Lisbon, Portugal, 14-17 June 2010.

${ }^{23}$ Lukashevich, S.V., Maslov, A.A., Shiplyuk, A.N., Fedorov, A.V., and Soudakov, V.G., "Stabilization of High-Speed Boundary Layer Using Porous Coatings of Various Thicknesses,” AIAA-2010-4720, 28 June - 1 July 2010, Chicago, Illinois.

${ }^{24}$ Colonius, T. and Lele, S., “Computational Aeroacoustics: Progress in Nonlinear Problems of Sound Generation,” Progress in Aerospace Sciences, Vol. 40, No. 6, 2004, pp. 345-416.

${ }^{25}$ Brès, G. A. and Colonius, T., “Three-Dimensional Instabilities in Compressible Flow over Open Cavities,” J. Fluid Mech., Vol. 599, 2008, pp. 309-339.

${ }^{26}$ Fedorov A.V., and Khokhlov, A.P., "Prehistory of Instability in a Hypersonic Boundary Layer,” Theoret. Comput. Fluid Dyn., Vol. 14, 2001, pp. 359-375.

${ }^{27}$ Fedorov, A., and Tumin, A., "Branching of Discrete Modes in High-Speed Boundary Layers and Terminology Issues,” AIAA-2010-5003, 28 June - 1 July 2010, Chicago, Illinois. 